\title{
A member of the Plasmodium falciparum PHIST family binds to the erythrocyte cytoskeleton component band 4.1
}

\author{
Lindsay A Parish ${ }^{1,2}$, Deborah W Mai ${ }^{1}$, Matthew L Jones ${ }^{2,3}$, Erika L Kitson ${ }^{2}$ and Julian C Rayner ${ }^{2,3^{*}}$
}

\begin{abstract}
Background: Plasmodium falciparum parasites export more than 400 proteins into the cytosol of their host erythrocytes. These exported proteins catalyse the formation of knobs on the erythrocyte plasma membrane and an overall increase in erythrocyte rigidity, presumably by modulating the endogenous erythrocyte cytoskeleton. In uninfected erythrocytes, Band 4.1 (4.1R) plays a key role in regulating erythrocyte shape by interacting with multiple proteins through the three lobes of its cloverleaf-shaped N-terminal domain. In P. falciparum-infected erythrocytes, the C-lobe of 4.1R interacts with the $P$. falciparum protein mature parasite-infected erythrocyte surface antigen (MESA), but it is not currently known whether other $P$. falciparum proteins bind to other lobes of the 4.1R $\mathrm{N}$-terminal domain.

Methods: In order to identify novel 4.1R interacting proteins, a yeast two-hybrid screen was performed with a fragment of 4.1R containing both the $\mathrm{N}$ - and a-lobes. Positive interactions were confirmed and investigated using site-directed mutagenesis, and antibodies were raised against the interacting partner to characterise it's expression and distribution in P. falciparum infected erythrocytes.
\end{abstract}

Results: Yeast two-hybrid screening identified a positive interaction between the 4.1R N-and a-lobes and PF3D7_0402000. PF3D7_0402000 is a member of a large family of exported proteins that share a domain of unknown function, the PHIST domain. Domain mapping and site-directed mutagenesis established that it is the PHIST domain of PF3D7_0402000 that interacts with 4.1R. Native PF3D7_0402000 is localized at the parasitophorous vacuole membrane (PVM), and colocalizes with a subpopulation of 4.1R.

Discussion: The function of the majority of $P$. falciparum exported proteins, including most members of the PHIST family, is unknown, and in only a handful of cases has a direct interaction between $P$. falciparum-exported proteins and components of the erythrocyte cytoskeleton been established. The interaction between 4.1R and PF3D7_0402000, and localization of PF3D7_0402000 with a sub-population of 4.1R at the PVM could indicate a role in modulating PVM structure. Further investigation into the mechanisms for 4.1R recruitment is needed.

Conclusion: PF3D7_0402000 was identified as a new binding partner for the major erythrocyte cytoskeletal protein, 4.1R. This interaction is consistent with a growing body of literature that suggests the PHIST family members function by interacting directly with erythrocyte proteins.

\footnotetext{
* Correspondence: julian.rayner@sanger.ac.uk

${ }^{2}$ William C Gorgas Center for Geographic Medicine, Division of Infectious

Diseases, Department of Medicine, University of Alabama at Birmingham, 845

19th St. South, Birmingham, AL 35294-2170, USA

${ }^{3}$ Malaria Programme, Wellcome Trust Sanger Institute, Wellcome Trust

Genome Campus, Hinxton, Cambridge CB10 1SA, UK

Full list of author information is available at the end of the article
} 


\section{Background}

Intraerythrocytic Plasmodium falciparum parasites extensively remodel their host cells. As the parasite matures the erythrocyte becomes more rigid, loses its biconcave shape for a more spherocytic one, and small electron dense protrusions, known as knobs, form on the surface [1]. These modifications enable the infected erythrocyte to adhere to and block the microvasculature and microcapillaries, which in turn contributes to specific complications such as cerebral and placental malaria [2,3]. Plasmodium falciparum achieves these massive changes in erythrocyte structure by exporting proteins into the erythrocyte cytosol, some of which interact with components of the host cytoskeleton and plasma membrane [3]. Only a few of these exported proteins have been studied in detail, such as knobassociated histidine rich protein (KAHRP), an essential structural component of knobs that secure the major cytoadherence ligand $P$. falciparum erythrocyte membrane protein 1 (PfEMP1) to the erythrocyte cytoskeleton through interactions with spectrin and actin [4-6], and mature parasite infected erythrocyte surface antigen (MESA), which binds to the erythrocyte cytoskeleton associated protein Band 4.1, also known as 4.1R [7,8].

For the majority of the more than $400 \mathrm{P}$. falciparum proteins that contain an export motif, referred to as the Plasmodium Export Element (PEXEL), little is know about their function $[3,9,10]$. This includes a group of 71 exported proteins that share a conserved domain of four alpha helices in tandem, termed the Plasmodium helical interspersed subtelomeric (PHIST) domain. PHIST domain-containing proteins can subdivided into three groups, PHISTa, PHISTb, and PHISTc, according to the position of one or two conserved tryptophans in the PHIST domain [11]. Several genes encoding PHIST proteins have been knocked out as part of a large-scale screen for exported protein function, and some of these knockouts showed a decrease in erythrocyte rigidity, implying that at least some PHIST proteins may play a role in modulating the erythrocyte cytoskeleton [12]. One PHISTb family member, ring infected surface antigen (RESA), is known to interact directly with the erythrocyte cytoskeleton by binding to spectrin tetramers, resulting in increased resistance to shear stress and thermal damage [13], while another binds to the C-terminal tail of PfEMP1 [14] but in the majority of cases no binding partner has been identified.

4.1R is involved in maintaining the biconcave shape, elasticity, and mechanical stability of human erythrocytes, and defects in 4.1R are one cause of hereditary erythrocyte elliptocytosis [15,16]. 4.1R is a member of a family of proteins that contain an N-terminal FERM domain, which regulates local cell shape by acting as a coordinator for protein-protein interactions [17]. Other FERM domain containing proteins, such as Ezrin, Radixin and Moesin, cross-link actin to the cytoplasmic tails of CD43, CD44, and ICAM-1 and have been implicated in regulation of cellular protrusions such as microvilli $[18,19]$. The 4.1R FERM domain interacts with erythrocyte membrane proteins Band 3, Glycophorin C and p55 through its $\mathrm{N}$-, $\alpha$ - and C-lobes, respectively, linking them to the actinspectrin network, which is bound by the 4.1R C-terminal domain [20-22].

Given the critical regulatory role that the 4.1R FERM domain plays in uninfected erythrocytes, it is not surprising that $P$. falciparum proteins also bind to it, and the C-lobe of the 4.1R FERM domain is recognized by MESA [23,24]. To test the hypothesis that the other lobes of the 4.1R FERM domain are also bound by exported $P$. falciparum proteins, a yeast two-hybrid screen of a $P$. falciparum cDNA library was performed using the $\mathrm{N}$ - and $\alpha$ - lobes of the 4.1R FERM domain as bait. This fragment of 4.1R interacted with PF3D7_0402000, an uncharacterized member of the PHISTa family. The 4.1R-PF3D7_0402000 interaction was mapped to the single amino acid level using the yeast two-hybrid system and visualized by immunofluorescence in P. falciparum-infected erythrocytes.

\section{Methods}

\section{Plasmodium falciparum culture}

Plasmodium falciparum 3D7 strain parasites were cultured in $\mathrm{O}+$ human erythrocytes and serum (purchased from Interstate Blood Bank) as described previously [25] and synchronized by application of $5 \%$ sorbitol during the ring stages. Use of human erythrocytes and serum for culture of P. falciparum was approved by University of Alabama at Birmingham Institutional Review Board.

\section{Yeast two-hybrid screen}

The Matchmaker GAL4 Two-Hybrid System 3 (Clontech) was used for yeast two-hybrid screens and analysis. A $P$. falciparum cDNA library containing activation domain fusions with fragments of $P$. falciparum proteins was kindly provided by Dr Lawrence Bergman (Drexel University). The 4.1R bait plasmid was made by cloning a fragment of the FERM domain of 4.1R (forward primer $5{ }^{\prime}$-GGAATTCA TGCACTGCAAGGTTTCTTTGTTGG-3', reverse primer 5' - CGGGATCCTTAATGAAGATCAACTCCATAC-3') into pGBKT7. The bait plasmid was transformed into yeast strain AH109, which was subsequently transformed with the $P$. falciparum cDNA-activation domain fusion library. Screening was performed by plating double transformants onto low stringency plates (-Leu/-Trp/-His) then replica plating onto high stringency plates (-Leu/-Trp/-His/-Ade) after four days growth. Activation domain plasmids were then rescued from all colonies that grew at high stringency, and retransformed into the original bait strain, as well as a strain transformed with an empty pGBKT7 vector, to test for plasmid independence and auto-activation. 
Yeast two-hybrid interaction validation and mapping

In order to recapitulate the interaction observed in the yeast-two-hybrid screen, a fragment of PF3D7_0402000 was cloned into pGADT7 (forward primer 5' - GGAATTC GGTAATAAGGAAGATAACCAGG-3', reverse primer 5' GGGGATCCTTAACCTCCTTGTTTTATATTTTGC-3'). Once the 4.1R-PF3D7_0402000 interaction was confirmed, a series of deletion and point mutation constructs of PF3D7_0402000 were made. The positions of the deletions and mutations are described in the text, and were made using PCR primers as follows: $\triangle$ ND1 construct (forward primer 5'-GGAATTCGAAAAGATATGGAATC ATGCCG-3', reverse primer 5' - GGGGATCCTTAACCTC CTTGTTTTATATTTTGC-3'), $\triangle$ CD1 construct (forward primer 5' - GGAATTCGGTAATAAGGAAGATAACC AGG-3', reverse primer 5' -GTGGATCCTTAAGTATAAT CATTTTCATGAAC-3'), $\triangle \mathrm{ND} 3$ (forward primer 5'-GGA ATTCAAAGAACAAATGAAAAATTTAG-3', reverse primer 5'-GGGGATCCTTAACCTCCTTGTTTTATATT TTGC-3), PHIST (forward primer 5' - GGGAATTCAT GTTAAGTCAGGACTATAATGAT-3' reverse primer 5' -GGGGATCCTTAGTCTATAGGAATTTCCGTAG-3'). Subfragments of the PF3D7_0402000 PHIST domain were created as follows: PHIST $\triangle \mathrm{C} 1$ (forward primer 5 '-GGGA ATTCATGCCCCGAAACGATATGGAAAAGATATGG-3', reverse primer 5'-GGGGATCCTTAGTCTATAGGAA TTTCCGTAG-3'), PHIST $\triangle$ C4 (forward primer 5' - GGGA ATTCATGTTAAGTCAGGACTATAATGAT-3' , reverse primer 5'-GGGGATCCTTAAGGGTCTTTATCAAGTAG TTC-3'), PHIST $\triangle C 1 C 4$ (forward primer 5'-GGGAATT CATGCCCCGAAACGATATGGAAAAGATATGG-3', reverse primer 5' -GGGGATCCTTAAGGGTCTTTATCA AGTAGTTC-3'). Mutation of the conserved W residue within the PF3D7_0402000 PHIST domain was completed by PCR sewing (forward primer 5'GGGAATTCATGCAG TTAACAAAAGAAGAATTATATG-3', and reverse primer 5'-GGGGATCCTTAGTCTATAGGAATTTCCGTAG-3', internal forward primer 5' - AACGATATGGAAAAGATA GCTAATCATGCCGTTAAAACA-3' and internal reverse primer 5' - TGTTTTAACGGCATGATTAGCTATCTTTT CCATATCGTT-3'). The PHIST domain from another PHIST family member was also cloned into pGADT7 as a control, using primers as follows: PF3D7_1253100 (forward primer 5' - GGGAATTCATGTTAAGTCAGGACTATAAT GAT-3', reverse primer 5'-GGGGATCCTTATTTTAATG AAAGAGTGTCC-3').

All PF3D7_0402000 and PF3D7_1253100 constructs were cloned into pGADT7 and tested for interaction with the 4.1R bait plasmid in yeast strain AH109 at both medium (-Leu/-Trp/-His plates) and high stringency (-Leu/-Trp/-His/-Ade). Critical interactions were quantified by transforming the 4.1R bait and PF3D7_0402000 interacting plasmid into yeast strain Y187 (Clontech) and measuring $\beta$-galactosidase activity using the Pierce yeast $\beta$-galactosidase assay kit as directed by manufacturer instructions. All $\beta$-galactosidase assays were performed in triplicate.

\section{Expression of 4.1R and PF3D7_0402000 for production of polyclonal antisera}

A fragment of human 4.1R was cloned from cDNA into the expression vector pRSETB (forward primer 5' -GAAGA TCTATGCACTGCAAGGTTTCTTTGTTGG-3', reverse 5' -CGGGATCCTTAATGAAGATCAACTCCATAC-3') and a fragment of PF3D7_0402000 was cloned into pRSETA (forward 5' -CGGGATCCGGATAAATATGAAGGATATG CTGC-3', reverse 5'-AACTGCAGTTAACCTCCTTGTT TTATATTTTGC-3'). Both plasmids were transformed into Escherichia coli BL21-DE3 pLysS. Recombinant proteins were expressed as hexa-his fusions and purified using either a Hi-Trap Chelating HP column (Amersham Biosciences) for the 4.1R fragment, or Nickel-NTA affinity agarose beads (Qiagen) for the PF3D7_0402000 fragment. The purified recombinant protein was then used to immunize both rabbits and rats (Cocalico Biologicals, Inc). PF3D7_0402000 rabbit antisera was affinity purified by coupling the purified hexa-his fusion protein to Hi-Trap NHS-activated beads (Amersham Biosciences), and passing the rabbit antisera over a column containing the NHSactivated beads. After extensive washing with $1 \mathrm{X}$ PBS, affinity-purified antibodies were eluted from the column with low pH buffer (0.1 M glycine, $0.5 \mathrm{M} \mathrm{NaCl}, \mathrm{pH} 2.5-2.8)$ directly into a neutralization buffer (0.1 M Tris, $\mathrm{pH} 8.8)$ and dialysed for 24 hours against $1 \times$ PBS.

\section{SDS PAGE and Western blots}

Parasite SDS lysates were made by resuspending $P$. falciparum-infected erythrocytes $\left(1-3 \times 10^{8}\right.$ parasites $\left./ \mathrm{ml}\right)$ in a $1 \times$ SDS sample buffer with $5 \% \beta$-mercaptoethanol. Triton X100 extracts were made by resuspending $P$. falciparum infected erythrocytes $\left(3 \times 10^{8}\right.$ parasites $\left./ \mathrm{ml}\right)$ in an equal volume of $1 \%$ Triton X100 in PBS. After centrifugation, the supernatant was saved as the Triton soluble fraction and the pellet was washed three times in 1\% Triton X100 in PBS. The pellet was resuspended in a $1 \times$ SDS sample buffer with $5 \% \beta$-mercaptoethanol and labelled as the Triton X100 insoluble fraction. SDS lysates or Triton X100 extracts were loaded onto and run on $12 \%$ polyacrylamide gels and then transferred to nitrocellulose membranes. Membranes were probed with primary antibodies as described, followed by HRP-conjugated secondary sera (anti-rabbit or anti-rat), and visualized using enhanced chemoluminescence (ECL, Amersham Biosciences).

\section{Immunofluorescence}

Air-dried thin smears of $P$. falciparum were either directly probed with anti-4.1R, anti-PF3D7_0402000, and/or 
PfSERA5, or were fixed with an ice-cold 50\% acetone/ $50 \%$ methanol solution and washed with $1 \times$ PBS before probing with the appropriate primary antisera. Secondary antisera (Molecular Probes) labeled with a fluorophore were used for visualization on an Olympus BX60 fluorescence microscope equipped with a Diagnostic Instruments Spot RT monochrome camera. All images were merged with Adobe Photoshop 5.0. Anti-PfSERA5 [26] was a kind gift from Dr Brendan Crabb.

\section{Results}

The 4.1R FERM domain interacts with PF3D7_0402000 in a yeast two-hybrid screen

A fragment of the 4.1R FERM domain containing the $\mathrm{N}$ - and $\alpha$-lobes was used as the bait in a yeast two-hybrid screen of a $P$. falciparum cDNA library (a kind gift from Dr Lawrence Bergman, Drexel University). Screening was performed in the yeast strain AH109 and transformants were originally plated at low stringency (-Leu/-Trp/-His), then transferred to high stringency plates (-Leu/-Trp/His/-Ade) after four days of growth. More than $1 \times 10^{6}$ colonies were screened and only nine grew on high stringency -Leu/-Trp/-His/-Ade plates. The plasmids from all nine positive colonies were isolated and retransformed with either the original $4.1 \mathrm{R}$ bait plasmid or an empty binding domain vector, pGBKT7, to test for plasmid independence and auto-activation. Two of the nine plasmids isolated from the screen did not recapitulate the growth on -Leu/-Trp/-His/-Ade plates and another three recapitulated the phenotype with the empty pGBKT7 vector, suggesting they were causing auto-activation.

The remaining four plasmids all recapitulated the phenotype of growth on high stringency plates in combination with the $4.1 \mathrm{R}$ bait plasmid, but not with an empty binding domain vector. Sequencing these plasmids revealed that all four contained fragments of the same P. falciparum gene, PF3D7_0402000. Three of the four plasmids contained identical inserts (Library 1; Figure 1), suggesting the same library clone was isolated independently three times, while the final plasmid contained a larger fragment of PF3D7_0402000 that overlapped in part with the other three plasmids (Library 2; Figure 1). To confirm the interaction a fragment of PF3D7_0402000 similar to library plasmid 1, but lacking six amino acids at the N-terminus, was amplified from genomic 3D7 DNA and cloned into pGADT7 (PF3D7_0402000; Figure 1). This fragment also supported growth on high stringency in combination with the $4.1 \mathrm{R}$ bait plasmid. The isolation of two overlapping fragments of PF3D7_0402000 in a yeast two-hybrid screen and the recapitulation of the interaction with an independent fragment of PF3D7_0402000 is strong evidence that the product of this gene interacts with the FERM domain of 4.1R.

\section{PF3D7_0402000 interacts with 4.1R through its PHIST domain}

PF3D7_0402000 encodes a 50kDa protein that is predicted to contain a hydrophobic TMD/anchor sequence, a $P$. falciparum export motif, and a PHIST domain (Figure 1). PHIST domains define a family of $71 P$. falciparum-exported proteins and consist of four tandemly arrayed alpha helices. The presence and location of two conserved tryptophan residues within the PHIST domain has been used to subdivide the PHIST family into three subfamilies. PF3D7_0402000 is a member of the PHISTa sub-family, which contains 23 members and unlike other PHIST subfamilies appears to be $P$. falciparum specific [11]. Following the PF3D7_0402000 PHIST domain is a series of repeated low-complexity regions at the C-terminal end, which is absent from both interacting plasmids isolated from the library.

In order to define the minimal domain of PF3D7_0402000 that interacts with $4.1 \mathrm{R}$, a series of smaller fragments of PF3D7_0402000 were cloned into pGADT7 and tested for interaction with the 4.1R bait vector on high stringency plates. When a portion of the C-terminal end of the library plasmid 1 was deleted, $(\Delta C)$, no growth was observed on Leu/-Trp/-His/-Ade- plates, suggesting this region is necessary for the PF3D7_0402000/4.1R interaction (amino acids 249-325). Deletion of an N-terminal fragment from the same plasmid, $(\Delta \mathrm{N} 1)$, also inhibited interaction with $4.1 \mathrm{R}$, whereas a smaller $\mathrm{N}$-terminal deletion, $(\Delta \mathrm{N} 3)$, allowed growth on both -Leu/-Trp/-His/and -Leu/-Trp/His/-Ade plates, suggesting that this region is dispensable for interaction with 4.1R (Figure 1).

These preliminary deletions suggested that it may be the PHIST domain of PF3D7_0402000 that interacts with $4.1 \mathrm{R}$, as the PHIST domain is largely preserved in all the deletion constructs that grew on high stringency plates. To test this hypothesis, the entire PHIST domain of PF3D7_0402000 (amino acids 160-300) was cloned into pGADT7 and tested for interaction with the 4.1R-bait plasmid. The PF3D7_0402000 PHIST domain alone was sufficient for interaction with $4.1 \mathrm{R}$, as growth was observed on both -Leu/-Trp/-His and -Leu/-Trp/-His/-Ade plates (PF3D7_0402000-PHIST; Figure 1). The interaction between the PF3D7_0402000 PHIST domain and 4.1R is specific, as the PHIST domain from another PHISTa family member, PF3D7_1253100, was not able to interact with the 4.1R bait plasmid (not shown).

\section{The conserved tryptophan in the PF3D7_0402000 PHIST domain is necessary for interaction with $4.1 \mathrm{R}$} The PHIST domain is defined largely by conserved structural predictions (four alpha helices in tandem of consistent sizes) rather than primary sequence conservation [11]. Alignment of the amino acid sequences of PHISTa domains reinforces this point, and suggests that the 


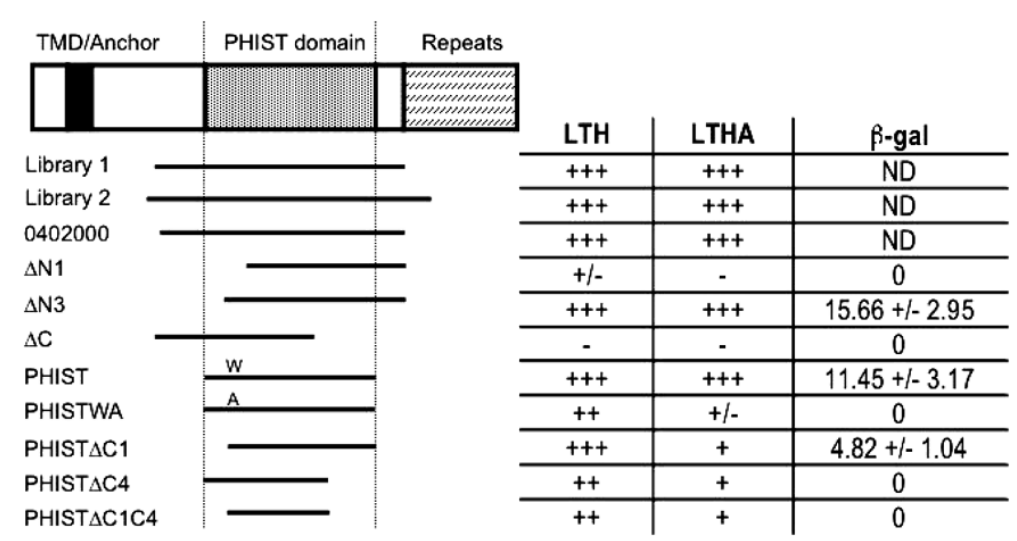

Figure 1 The PF3D7_0402000 PHIST domain interacts with 4.1R. Cartoon of PF3D7_0402000 showing the predicted transmembrane domain (TMD) or signal anchor, the PHISTa domain, and the C-terminal repeats. The regions of PF3D7_0402000 that were present in the rescued P. falciparum CDNA library plasmids (Library 1 and 2), or subsequently amplified and cloned into the yeast two-hybrid vector pGADT7 are indicated below the cartoon. The ability of each construct to interact with the 4.1R bait was scored at low (LTH: growth on -Leu/-Trp/-His plates) and high stringency (LTHA: growth on -Leu-/Trp/-His/-Ade plates) in strain AH109. Selected positive constructs were quantitatively scored for $\beta$-galactosidase activity assays in strain Y187 (assays performed in triplicate; data presented is the average $+/$ - standard deviation between replicates).

consensus PHISTa domain consists of two semi-conserved domains linked by a poorly conserved spacer fragment that in several proteins has one or more helix-breaking proline residues (see Figure 2). Some of the smaller PHISTa domains contain only the $\mathrm{N}$-terminal semi-conserved domain (PF3D7_1253900, for example), while others contain only the C-terminal semi-conserved domain (PF3D7_1149700, for example). As previously reported, among the most conserved residues in the PHISTa domains are two tryptophans, one in each semi-conserved domain. Most PHISTa family members contain both tryptophan residues (see Figure 2, marked with closed circles at residues 26 and 81), but PF3D7_0402000 contains only the first one. To test the hypothesis that this conserved tryptophan residue is important for the interaction of PF3D7_0402000 with 4.1R, it was mutated to alanine in the PF3D7_0402000 PHIST domain construct. This point mutant allowed for growth on -Leu/-Trp/-His but only slow growth on -Leu/Trp/-His/-Ade PHISTWA; (Figure 1). To quantify the interaction, $\beta$-galactosidase activity was measured in the strain Y187, which is a more stringent test (see Methods). No activity was detected when PHISTWA and the 4.1R bait plasmid were co-expressed in Y187, indicating that the conserved tryptophan is needed for an efficient interaction between PF3D7_0402000 and 4.1R.

To test whether all four PHIST helices are necessary for PF3D7_0402000 binding to 4.1R, the first and fourth helices were deleted, both alone and together, from the PHIST binding domain construct. Deletion of either coil 1 or coil 4 of the PHIST domain individually (PHIST $\triangle \mathrm{C} 1$ and PHIST $\triangle \mathrm{C} 4$ ) or both helices together (PHIST $\triangle \mathrm{C} 1 \mathrm{C} 4)$, did not completely eliminate the interaction with $4.1 \mathrm{R}$ as all three constructs allowed growth on both -Leu/-Trp/-His and -Leu/-Trp/-His/-Ade plates
(Figure 1). However, when $\beta$-galactosidase assays were performed it became apparent that the full PHIST domain was needed for maximal interaction with 4.1R. Deletion of both coils 1 and 4 together, or coil 4 alone, completely eliminated $\beta$-galactosidase activity, while deletion of coil 1 had only minimal $\beta$-galactosidase activity (Figure 1 ). Taken together, the results suggest that neither coil 1 nor coil 4 are absolutely required for interaction with $4.1 \mathrm{R}$, but the full PHIST domain is required for maximal interaction as determined by $\beta$-galactosidase assays.

\section{PF3D7_0402000 is expressed at the parasitophorous vacuole membrane}

To determine the localization of endogenous PF3D7_ 0402000 in infected erythrocytes, both rabbit and rat polyclonal antibodies were raised against a purified hexa histidine-tagged fragment of PF3D7_0402000 that spans from the end of the putative transmembrane, representing amino acids 51-325 of the predicted protein sequence. Western blots of $P$. falciparum extracts probed with the resulting rat anti-PF3D7_0402000 antibody predominantly identified a single band at approximately $60 \mathrm{kDa}$, larger than the predicted size of $50 \mathrm{kDa}$, or of $41 \mathrm{kDa}$ for a PEXEL processed form of PF3D7_0402000 (Figure 3A). One possible explanation for the unexpected mobility is that the antibodies are primarily recognizing a form of PF3D7_0402000 where PEXEL cleavage has not occurred. This would seem to be unlikely, however, because the PEXEL cleavage site is at amino acid residue 72 , meaning that only 15 amino acids of sequence prior to the predicted cleavage site are present in the recombinant protein used to generate the polyclonal antibodies, compared to 259 amino acids of the predicted mature protein. However, the PF3D7_0402000 protein sequence 


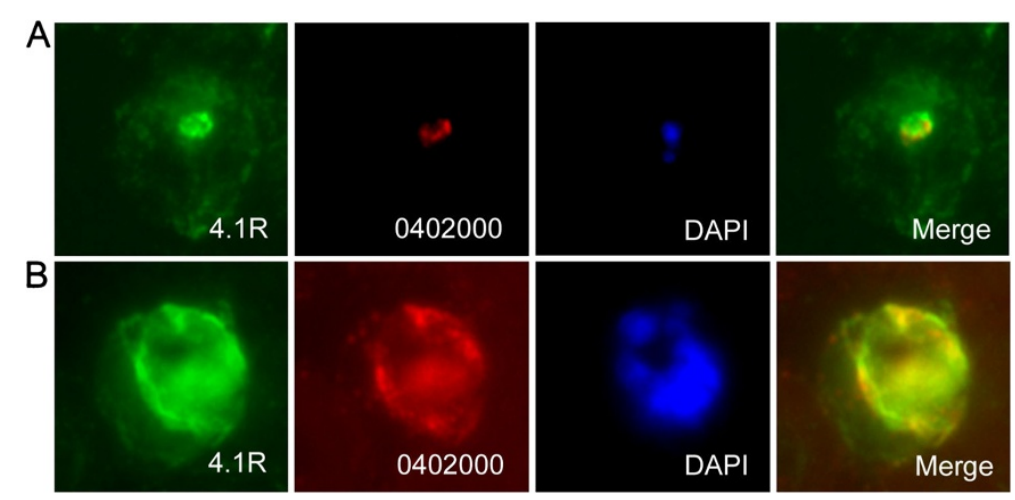

Figure 4 PF3D7_0402000 colocalizes with a sub-population of 4.1R. Immunofluorescence microscopy of air-dried smears of 3D7 P. falciparum-infected erythrocytes probed with rabbit anti-4.1R (green), rat anti-PF3D7_0402000 (red) and the nuclear dye (DAPI). PF3D7_0402000 and 4.1R colocalize in early (A) and late (B) trophozoite stage parasites.

does contain an extended unstructured region, and such regions are known to cause aberrant mobility of $P$. falciparum proteins in SDS-PAGE.

Expression of PF3D7_0402000 was detected in trophozoite and schizont extracts, but not early ring stage extracts or in uninfected erythrocytes (Figure 3A), consistent with published $P$. falciparum microarray data, which show peak expression of PF3D7_0402000 in 3D7 at approximately 24 hours [29,30]. To determine the intracellular location of PF3D7_0402000, affinity-purified anti-PF3D7_0402000 antibodies were used in immunofluorescence experiments. Air-dried smears of 3D7 parasites were co-stained with specific antibodies against PF3D7_0402000 and PfSERA5. A significant portion of PF3D7_0402000 colocalized with PfSERA5 (Figure 3B), suggesting that PF3D7_0402000 is a PVM protein.
A sub-population of 4.1R colocalizes with PF3D7_0402000 In order to confirm that 4.1R and PF3D7_0402000 interact in vivo, co-immunoprecipitation experiments were attempted but were thwarted by the fact that $4.1 \mathrm{R}$ is detergent insoluble in both uninfected and infected erythrocytes (data not shown), making the recovery of detectable amounts of 4.1R from detergent extracts by immunoprecipitation impossible. However, co-immunofluorescence with antibodies against PF3D7_0402000 and 4.1R show that a subpopulation of $4.1 \mathrm{R}$ clearly colocalizes with PF3D7_0402000 at the PVM (Figure 4A, B). The presence of $4.1 \mathrm{R}$ at the PVM is not due to cross-reaction with $P$. falciparum proteins, as the anti-4.1R antisera recognizes exactly the same fragments in SDS extracts from uninfected (-) and P. falciparum infected (Pf) erythrocytes, with a major band at $80 \mathrm{kDa}$, the size of mature 4.1R (Figure $5 \mathrm{~A}$ ).
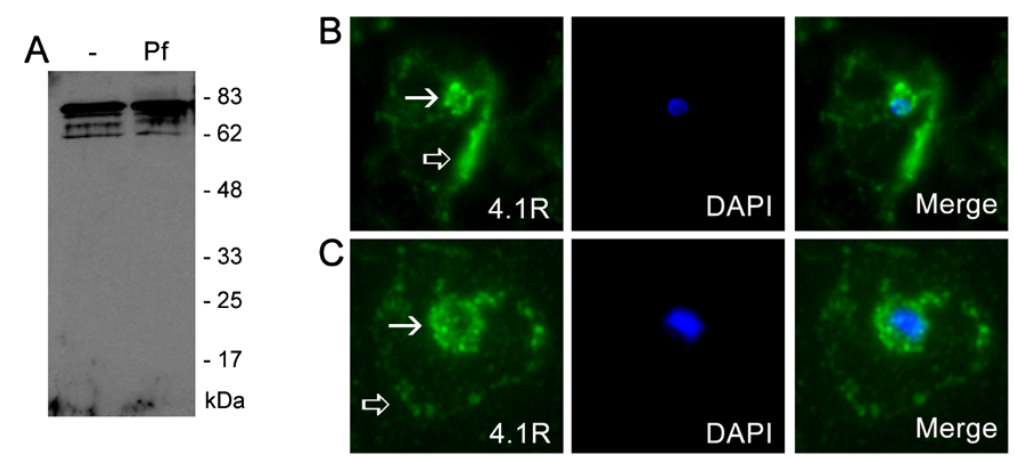

Figure 5 A subpopulation of 4.1R is found at the PVM in Plasmodium falciparum-infected erythrocytes. (A) Rabbit polyclonal antibodies raised against the FERM domain of $4.1 \mathrm{R}$ recognize a major band at the size of full-length $4.1 \mathrm{R}(80 \mathrm{kDa})$ in both uninfected (-) and $P$. falciparuminfected $(\mathrm{Pf})$ erythrocytes. Additional bands are either proteolytic cleavage products or alternatively spliced products of $4.1 \mathrm{R}$ as the same bands were recognized in both uninfected and P. falciparum-infected erythrocytes. (B, C) Immunofluorescence microscopy of air-dried smears of 3D7 P. falciparum-infected erythrocytes probed with rabbit anti-4.1R (green). 4.1R consistently localizes near the erythrocyte plasma membrane (black arrows), but in early (B) and late (C) trophozoite-stage parasites, a subpopulation of 4.1R is found in close apposition to the parasite, as detected by DAPI staining (blue). 
4.1R is by no means completely relocalized to the PVM in infected erythrocytes, as a significant proportion of $4.1 \mathrm{R}$ staining remains at the erythrocyte periphery (black arrow, Figure $5 \mathrm{~B}, \mathrm{C}$ ) consistent with the known interactions of $4.1 \mathrm{R}$ with the erythrocyte plasma membrane proteins glycophorin $\mathrm{C}$ and Band 3, and the P. falciparum protein MESA. However, some 4.1R is clearly found at the PVM, consistent with an interaction of a subpopulation of $4.1 \mathrm{R}$ with PF3D7_0402000.

\section{Discussion}

During the intraerythrocytic life cycle of P. falciparum, the parasite is predicted to export between 400-600 proteins into the erythrocyte cytosol, based on the presence of a motif (termed PEXEL) that is required for the export of many known exported proteins [9,10]. The function of many of these exported proteins is unknown. PfEMP1, KAHRP, and MESA are all P. falciparum-exported proteins that interact with the erythrocyte cytoskeleton and play a role in changing the structure of the erythrocyte membrane and cytoskeleton. The PHIST proteins, originally identified through searching the $P$. falciparum genome for PEXEL-containing proteins [11], may play a similar role, as knockouts of several PHIST family members show a decrease in erythrocyte rigidity [12]. In this study, one member of the PHIST family, PF3D7_0402000, was shown to interact with the FERM domain of $4.1 \mathrm{R}$ in a yeast two-hybrid screen. Formal confirmation of the interaction by co-immunoprecipitation was not possible due to the insolubility of $4.1 \mathrm{R}$, but it should be pointed out that a complex between MESA and 4.1R has not been co-immunoprecipitated from infected erythrocytes either, presumably for the same reason. However PF3D7_0402000 colocalizes with a subpopulation of 4.1R, supporting an interaction in vivo.

How could only a subpopulation of $4.1 \mathrm{R}$ be recruited to the PVM? Interestingly, increased phosphorylation of 4.1R has been observed in P. falciparum-infected erythrocytes, [31,32]. Phosphorylation of 4.1R has been shown to weaken its affinity for glycophorin $\mathrm{C}$, actin, and spectrin and takes place sometime between early ring and early trophozoite stages [33]. It is possible that phosphorylation, or some other post-translational modification event that occurs in P. falciparum-infected erythrocytes, divides 4.1R into two distinct populations: one that remains associated with erythrocyte cytoskeleton and one that dissociates from the erythrocyte cytoskeleton and is available to interact with PF3D7_0402000 at the PVM. This model is clearly hypothetical, but should be readily testable. Relocalization of erythrocyte cytoskeleton components to the PVM in $P$. falciparum-infected erythrocytes is not without precedent. Erythrocytes contain a class II myosin, which also appears to partially relocalize around the PVM (31). Moreover, it has been shown that $4.1 \mathrm{R}$ can bind to this class II myosin through its spectrin and actin-binding domain [34]. Thus, it is possible that relocalized 4.1R binds and interacts with relocalized myosin as well as PF3D7_0402000 at the PVM.

The PVM is a dynamic membrane, which expands as the parasite divides and increases in size inside the erythrocyte. Given the role of $4.1 \mathrm{R}$ in maintaining structure at the erythrocyte surface, the presence of both $4.1 \mathrm{R}$ and myosin at the PVM is clearly consistent with a role in maintaining the stability of the PVM during this expansion process. It is also possible that 4.1R at the PVM plays a role in protein transport. Both the tubovesicular network, which consists of threadlike projections of the PVM that extend into the erythrocyte cytosol, and the formation of Maurer's clefts, which may bud from the PVM $[35,36]$, are modifications of the PVM. Other FERM domain-containing proteins, such as ezrin, radixin and moeisin, promote cellular protrusions such as microvilli $[18,19]$. 4.1R could perform a similar function at the PVM and be involved directly in TVN and/or Maurer's cleft formation and budding, perhaps by cross-linking the PVM with other structural components, whether host or parasite derived. These hypothetical roles for 4.1R at the PVM remain to be tested.

The PHIST domain and PHIST family proteins were originally identified through bioinformatic approaches based on conserved structural features [10]. The identification of an interaction between the PHIST domain of PF3D7_0402000 and 4.1R allowed direct testing of whether the bioinformatic parameters used to define the PHIST families are functionally relevant. While neither the first or fourth helix or the conserved tryptophan residue in the PF3D7_0402000 PHISTa domain were absolutely necessary for interaction with 4.1R, the full PHIST domain and the conserved tryptophan were all required for a highly stringent interaction as measured by $\beta$-galactosidase activity. The broad outlines of the PHIST domain described by bioinformatics do therefore appear to have functional relevance. Knockout of several PHIST family members shows a decrease in erythrocyte rigidity [11]. Given the extensive remodelling of the erythrocyte cytoskeleton that occurs after infection by the P. falciparum parasite, it would seem reasonable to hypothesize that the PHIST domain has evolved to bind directly with an array of endogenous erythrocyte proteins, including components of the cytoskeleton, with individual PHIST domain proteins recognizing specific, and probably overlapping, targets. The PHIST family may therefore represent a central front in the parasitehost interactions that drive the successful colonization of human erythrocytes by $P$. falciparum parasites.

\section{Competing interests}

The authors declare that they have no competing interests.

\section{Authors' contributions}

$L P, J R, D M$, and MJ designed the experiments: LP, MJ, DM, and EK performed the experiments: LP, JR, DM, and MJ analysed the data: LP and JR wrote the paper. All authors read and approved the final manuscript. 


\section{Acknowledgements}

We thank Dr Brendan Crabb for the anti-PfSERA5 antibodies and Dr Lawrence Bergman for the P. falciparum CDNA yeast two-hybrid library. This work was supported by National Institutes of Health grant R03 Al064849.

\section{Author details}

${ }^{1}$ Department of Microbiology, University of Alabama at Birmingham, 845 19th St South, Birmingham, AL 35294-2170, USA. William C Gorgas Center for Geographic Medicine, Division of Infectious Diseases, Department of Medicine, University of Alabama at Birmingham, 845 19th St. South, Birmingham, AL 35294-2170, USA. ${ }^{3}$ Malaria Programme, Wellcome Trust Sanger Institute, Wellcome Trust Genome Campus, Hinxton, Cambridge CB10 1SA, UK

Received: 2 March 2013 Accepted: 30 April 2013

Published: 11 May 2013

\section{References}

1. Nash GB, O'Brien E, Gordon-Smith EC, Dormandy JA: Abnormalities in the mechanical properties of red blood cells caused by Plasmodium falciparum. Blood 1979, 74:855-861

2. Hanssen E, MicMillan PJ, Tilley L: Cellular architecture of Plasmodium falciparum-infected erythrocytes. Int J Parasitol 2010, 40:1127-1135.

3. Maier AG, Cooke BM, Cowman AF, Tilley L: Malaria parasite proteins that remodel the host erythrocyte. Nat Rev Micro 2009, 7:341-354.

4. Crabb BS, Cooke BM, Reeder JC, Waller RF, Caruana SR, Davern KM, Wickham ME, Brown GV, Coppel RL, Cowman AF: Targeted gene disruption shows that knobs enable malaria-infected red cells to cytoadhere under physiological shear stress. Cell 1997, 89:287-296.

5. Waller KL, Cooke BM, Nunomura W, Mohandas N, Coppel RL: Mapping the binding domains involved in the interaction between the Plasmodium falciparum knob-associated histidine-rich protein (KAHRP) and the cytoadherence ligand $P$. falciparum erythrocyte membrane protein 1 (PfEMP1). J Biol Chem 1999, 274:23808-23813.

6. Oh SS, Voigt S, Fisher D, Yi SJ, LeRoy PJ, Derick LH, Liu S, Chishti AH: Plasmodium falciparum erythrocyte membrane protein 1 is anchored to the actin-spectrin junction and knob-associated histidine-rich protein in the erythrocyte skeleton. Mol Biochem Parasitol 2000, 108:237-247.

7. Lustigman S, Anders RF, Brown GV, Coppel RL: The mature-parasite -infected erythrocyte surface antigen (MESA) of Plasmodium falciparum associates with the erythrocyte membrane skeletal protein, 4.1R. Mol Biochem Parasitol 1990, 38:261-270

8. Magowan C, Coppel RL, Lau AO, Moronne MM, Tchernia G, Mohandas N: Role of the Plasmodium falciparum mature-parasite-infected erythrocyte surface antigen (MESA/PfEMP-2) in malarial infection of erythrocytes. Blood 1995, 86:3196-3204.

9. Hiller NL, Bhattacharjee S, Van Ooij C, Liolios K, Harrison T, Lopez-Estrano C, Haldar K: A host-targeting signal in virulence proteins reveals a secretome in malarial infection. Science 2004, 306:1934-1937.

10. Marti M, Good RT, Rug M, Knuepfer E, Cowman AF: Targeting malaria virulence and remodeling proteins to the host erythrocyte. Science 2004, 306:1930-1933.

11. Sargeant TJ, Marti M, Caler E, Carlton JM, Simpson K, Speed TP, Cowman AF: Lineage-specific expansion of proteins exported to erythrocytes in malaria parasites. Genome Biol 2006, 7:R12.

12. Maier AG, Rug M, O’Neill MT, Brown M, Chakravorty S, Szestak T, Chesson J, Wu Y, Hughes K, Coppel RL, Newbold C, Beeson JG, Craig A, Crabb BS, Cowman AF: Exported proteins required for virulence and rigidity of Plasmodium falciparum-infected human erythrocytes. Cell 2008, 134:48-61

13. Pei X, Guo X, Coppel R, Bhattacharjee S, Haldar K, Gratzer W, Mohandas N, An $X$ : The ring-infected erythrocyte surface antigen (RESA) of Plasmodium falciparum stabilizes spectrin tetramers and suppresses further invasion. Blood 2007, 110:1036-1042.

14. Mayer C, Slater L, Erat MC, Konrat R, Vakonakis I: Structural analysis of the Plasmodium falciparum Erythrocyte Membrane Protein 1 (PfEMP1) intracellular domain reveals a conserved interaction epitope. $J \mathrm{Bio} / \mathrm{Chem}$ 2012, 287:7182-7189.

15. Diakowski W, Grzybek M, Sikorski AF: Protein 4.1, a component of the erythrocyte membrane skeleton and its related homolog proteins forming the protein 4.1/FERM superfamily. Folia Histochem Cytobio/ 2006, 44:231-248.
16. Tchernia G, Mohandas N, Shohet SB: Deficiency of skeletal membrane protein 4.1R in homozygous hereditary elliptocytosis. Implications for erythrocyte membrane stability. J Clin Invest 1981, 68:454-460.

17. Chishti AH, Kim AC, Marfatia SM, Lutchman M, Hanspal M, Jindal H, Liu SC, Low PS, Rouleau GA, Mohandas N, Chasis J, Conboy JG, Gascard P, Takakuwa Y, Huang SC, Benz EJ Jr, Bretscher A, Fehon RG, Gusella JF, Ramesh V, Solomon F, Marchesi VT, Tsukita S, Tsukita S, Hoover KB: The FERM domain: a unique module involved in the linkage of cytoplasmic proteins to the membrane. Trends Biochem Sci 1998, 23:281-282.

18. Yonemura S, Hirao M, Doi Y, Takahashi N, Kondo T, Tsukita S, Tsukita S: Ezrin/radixin/moesin (ERM) proteins bind to a positively charged amino acid cluster in the juxta-membrane cytoplasmic domain of CD44, CD43, and ICAM-2. J Cell Biol 1998, 140:885-895.

19. Yonemura S, Tsukita S, Tsukita S: Direct involvement of ezrin/radixin/moesin (ERM)-binding membrane proteins in the organization of microvilli in collaboration with activated ERM proteins. J Cell Biol 1999, 145:1497-1509.

20. Nunomura W, Takakuwa Y, Parra M, Conboy J, Mohandas N: Regulation of protein $4.1 \mathrm{R}$, p55, and glycophorin $\mathrm{C}$ ternary complex in human erythrocyte membrane. J Biol Chem 2000, 275:24540-24546.

21. Bong-Gyoon H, Nunomura W, Takakuwa Y, Mohandas N, Jap BK: Protein 4.1R core domain structure and insights into the regulation of cytoskeletal organization. Nature Struct Biol 2000, 7:871-875.

22. Schischmanoff PO, Winardi R, Discher DE, Parra MK, Bicknese SE, Witkowska $\mathrm{HE}$, Conboy JG, Mohandas N: Defining of the minimal domain of protein 4.1 involved in spectrin-actin binding. J Biol Chem 1995, 270:21243-21250.

23. Waller KL, Nunomura W, An X, Cooke BM, Mohandas N, Coppel RL: Mature parasite-infected erythrocyte surface antigen (MESA) of Plasmodium falciparum binds to the $30-\mathrm{kDa}$ domain of protein 4.1 in malaria infected-red blood cells. Blood 2003, 102:1911-1914.

24. Black CG, Proellocks NI, Kats LM, Cooke BM, Mohandas N, Coppel RL: In vivo studies support the role of trafficking and cytoskeletal-binding motifs in the interaction of MESA with the membrane skeleton of Plasmodium falciparum infected red blood cells. Mol Biochem Parasitol 2008, 160:143-147.

25. Trager W, Jensen JB: Human malaria parasites in continuous culture. Science 1979, 193:673-676.

26. Miller SK, Good RT, Drew DR, Delorenzi M, Sanders PR, Hodder AN, Speed TP, Cowman AF, De Koning-Ward TF, Crabb BS: A subset of Plasmodium falciparum SERA genes are expressed and appear to play an important role in the erythrocytic cycle. J Biol Chem 2002, 277:47524-47553.

27. Edgar RC: MUSCLE: multiple sequence alignment with high accuracy and high throughput. Nucleic Acids Res 2004, 32:1792-1797.

28. Waterhouse AM, Procter JB, Martin DMA, Clamp M, Barton GJ: Jalview Version 2 - a multiple sequence alignment editor and analysis workbench. Bioinformatics 2009, 25:1189-1191.

29. Bozdech Z, Llinás M, Pulliam BL, Wong ED, Zhu J, DeRisi JL: The transcriptome of the intraerythrocytic developmental cycle of Plasmodium falciparum. PLOS Biol 2003, 1:85-100.

30. Vega P, Holder AA, Batalov S, Carucci DJ, Winzeler EA: Discovery of gene function by expression profiling of the malaria parasite life cycle. Science 2003, 301:1503-1508.

31. Chishti AH, Maalouf GJ, Marfatia S, Palek J, Wang W, Fisher D, Liu SC: Phosphorylation of protein 4.1 in Plasmodium falciparum-infected human red blood cells. Blood 1994, 83:3339-3345.

32. Manno S, Takakuwa Y, Mohandas N: Modulation of erythrocyte membrane mechanical function by protein 4.1 phosphorylation. J Biol Chem 2005, 280:7581-7587.

33. Taraschi TF, O'Donnell M, Martinez S, Schneider T, Trelka D, Fowler VM, Tilley L, Moriyama Y: Generation of an erythrocyte vesicle transport system by Plasmodium falciparum malaria parasites. Blood 2003, 120:3420-3426.

34. Pasternack GR, Racusen RH: Erythrocyte protein 4.1 binds and regulates myosin. Proc Natl Acad Sci USA 1989, 86:9712-9716.

35. Spycher C, Rug M, Klonis N, Ferguson DJP, Cowman AF, Beck HP, Tilley L: Genesis of and trafficking to the Maurer's clefts of Plasmodium falciparum-infected erythrocytes. Mol Cell Biol 2006, 26:4074-4085.

36. Tilley L, Sougrat R, Lithgow T, Hansen E: The twists and turns of Maurer's Cleft Transport in Plasmodium falciparum-infected erythrocytes. Traffic 2008, 9:187-197.

doi:10.1186/1475-2875-12-160

Cite this article as: Parish et al: A member of the Plasmodium falciparum PHIST family binds to the erythrocyte cytoskeleton component band 4.1. Malaria Journal 2013 12:160. 